\title{
New Technique for Intrathoracic Implantation of Extraluminal Spiral Prostheses in the Trachea
}

\author{
Hércules Lúcio Gomes, Rodiney Pinheiro Denevitz, Isabella Cristina Morales, \\ Scarlath Ohana Penna dos Santos, Haroldo José Siqueira da Igreja Júnior, Marcelo Borges dos Santos Júnior, \\ Tainara Micaele Bezerra Peixoto, Jussara Peters Scheffer, Fernanda Antunes \& André Lacerda de Abreu Oliveira
}

\begin{abstract}
Background: Extraluminal surgical procedures for intrathoracic tracheal collapse in dogs are not routinely performed. The patients are normally treated with different drugs or by intraluminal stents. However, in more severe cases, drug treatment does not always have good outcomes, and intraluminal prostheses can be correlated to several postoperative problems. In order to obtain better results, we aimed to develop a surgical technique for implantation of a new extraluminal helical prosthesis in the thoracic segment of the trachea through cervical access, associated with pneumatic mediastinoscopy for certification of the technique and minimization of possible complications.

Materials, Methods \& Results: Seven canine corpses (CCs) from non-traumatic death, weighing between 2 and $7 \mathrm{~kg}$, were used. A ventral cervical approach to the trachea was associated with blunt mediastinal dissection. Trans cervical pneumatic mediastinoscopy was used for evaluation of the dissection and location of the implant. These were compared with the necropsy findings by the exact Wilcoxon two-sample test, with $P<0.05$. The results of necropsy and mediastinoscopy did not present significant differences at $P<0.05$. During the examinations, the presence of some mediastinal visceral lesions caused by the prosthesis, the integrity of the mediastinum and possible lesions to RLN and blood vessels (BV) were analyzed. We also investigated the location of the distal part of the prosthesis in the thoracic segment of the trachea and its dissection. To evaluate the technique, statistical comparison was made between mediastinoscopy and necropsy findings. The data were compared by the Wilcoxon test at 5\% probability. The tracheas of all CCs were efficiently dissected, but in some cases problems that can happen during the procedure were noticed. This was checked by mediastinoscopy and confirmed by necropsy. The median of the scores was 1 (good dissection and visualization), with variance of zero for mediastinoscopy and 0.14 for necropsy. In the mediastinoscopy of one $\mathrm{CC}$, the prosthesis crossed the tracheal bifurcation, involving the posterior vessels of this region, which was confirmed by necropsy. However, the dissection showed no vascular lesions. The integrity of the mediastinum was assessed only by mediastinoscopy. There was no impairment caused by any of the procedures performed. This resulted in a median score of 1 (integrity) with zero variance. When the positioning of the prosthesis was evaluated in relation to the tracheal bifurcation, identical values were obtained by both methods. The median score was 1 , with variance of 0.62 . No statistical differences were found between the two evaluation methods for the analyzed variables. This demonstrates the strong potential of the proposed evaluation techniques.

Discussion: Our results show that the execution of the technique is possible, but some complications may occur. Mediastinoscopy as an evaluation procedure can pose some complications, these considerations are important, but by using technique proposed in this study, these factors are minimized due to the ease of technical implantation of the extraluminal tracheal stent, without the need for several instruments competing in the inflated mediastinal space. We concluded that the technique is viable and a safe method with minimal invasion for investigation and treatment of mediastinal diseases, presenting low impairment of the paratracheal structures, and that mediastinoscopy can be used as a transoperative evaluation method to minimize complications.
\end{abstract}

Keywords: tracheal collapse, intrathoracic, pneumatic mediastinoscopy, prosthesis, video-assisted.

DOI: $10.22456 / 1679-9216.110187$ [lacerdavet@uol.com.br]. Department of Veterinary Surgery - UENF. Av. Alberto Lamego n. 2000. CEP 28013-602 Campos dos Goytacazes, RJ, Brazil. 


\section{INTRODUCTION}

Tracheal collapse (TC) is a chronic progressive respiratory disease characterized by dorsoventral flattening of the trachea due to the weakening of the cartilaginous rings and widening of the tracheal muscle, which can occur in the cervical, thoracic and cervicothoracic portion. It is a common disease in adult dogs, especially small breed dogs $[12,13]$. Clinical signs of TC include respiratory problems, such as dry and severe cough ("goose honk" cough), raspy breathing, and occasionally dyspneic episodes and cyanosis [13] which can lead to death from airway obstruction in severe and progressive cases [12].

Dogs with TC are initially treated with a combination of medicines, to control clinical signs and maintain quality of life. Currently, implantation of intraluminal or extraluminal stents of nitinol are the most common surgical techniques used for treatment. However, different complications have been reported, such as inflammatory process at the implantation site and tracheitis, granuloma formation, stent fracture or migration, and organ perforation $[9,14,15]$ and iatrogenic laryngeal paralysis in the immediate postoperative period $[1,3,14,15]$, respectively.

The intrathoracic approach for extraluminal correction is not easily performed [4,5] and it is correlated with high mortality and morbidity. We aimed to develop a surgical technique for implantation of a new extraluminal helical prosthesis in the thoracic segment of the trachea through cervical access, associated with pneumatic mediastinoscopy for certification of the technique and minimization of possible complications by applying the prosthesis without a thoracotomy approach.

\section{MATERIALS AND METHODS}

\section{Experimental animal selection}

Seven canine corpses (CCs) from nontraumatic death, weighing between 2 and $7 \mathrm{~kg}$, were used. Of these, 4 were males and 3 were females. They presented variable ages, as estimated by dental characteristics, classified as 3 juveniles, 2 adults and 2 elderly dogs.

\section{Experimental design and surgical technique}

The CCs were prepared to undergo the operative technique and intubated with an endotracheal tube, and then placed in dorsal decubitus with anterior limbs drawn caudally and positioned parallel to the thorax wall. A foam pad was placed below the dorsal cervical region and a bandage kept the muzzle parallel to the operating table, which provided a slight cervical ventroflexion, facilitating tracheal exposure.

A medial incision was made in the ventral region of the neck. With curved Metzenbaum scissors, the subcutaneous tissue and the medial raphe of the sternohyoid muscles were divided and the segments were moved away with two self-static Gelpi retractors, positioned in the proximal and distal portions of the neck, to expose the trachea.

After tracheal identification, two 2-0 nylon stay sutures were placed around a cranial tracheal ring to facilitate tracheal cranial traction. Then peritracheal tissue was dissected, forming a tunnel around trachea, and the trachea was raised with a Kelly clamp on its dorsal surface, facilitating dissection throughout the cervical portion. On its caudal portion, the surgeon used a Kelly clamp to perform blunt digital dissection of the thoracic trachea through the cranial mediastinum.

After dissection of the trachea, mediastinoscopy was performed with a $10 \mathrm{~mm}$ diameter rigid endoscope and a pneumomediastinum was created by carbon dioxide insufflation $(4 \mathrm{mmHg})$, which confirmed the quality of the tracheal dissection and enabled visualization of the tracheal bifurcation, making it possible to locate some segments that needed additional dissection.

A helicoidal nitinol prosthesis was positioned externally along the cervical trachea and rotational movement was performed in order to fit it all around the trachea. After implantation, the prosthesis was directed to the tracheal bifurcation with cranial sliding helicoidal movements to its caudal position. Prosthesis guidance was performed by the surgeon with his finger, who by synesthesia tried to properly locate the prosthesis near the tracheal bifurcation. At the end of the positioning, 2 simple perforating stitches were performed with 3-0 polypropylene thread to affix the implant to the trachea.

After prosthesis fixation, another mediastinoscopy was performed. This aimed to identify possible complications during implantation of the prosthesis, such as recurrent laryngeal nerve (RLN) lesions, mediastinal rupture, vascular lesions, incarceration of the esophagus or other peri-tracheal anatomical structures, as well as to confirm the positioning of the prosthesis in relation to the tracheal bifurcation. 


\section{Scores determination}

In order to evaluate the surgical technique, mediastinoscopy findings were compared to necropsy. For each analyzed variable, a numerical value of 1 or 2 was assigned, according to Table 1.

Seven variables were analyzed in relation to possible changes during the technique's performance, scoring 1 for presence and scoring 2 for absence: (1) trachea isolation along the path to the bifurcation without traumatic injuries during dissection; (2) injury and/ or imprisonment of the RLN by the prosthesis or dissection; (3) traumatic esophageal injury of perforation or imprisonment by the prosthesis; (4) vascular injury in the mediastinal space with involvement (rupture or imprisonment by the prosthesis) of any peri-tracheal vein or artery; (5) proper identification of tracheal bifurcation after digital mediastinal dissection; (6) mediastinal integrity during dissection and implantation of the prosthesis; and the location of the prosthesis in the tracheal bifurcation.

\section{Statistical analysis}

All variables were statistically compared by the exact Wilcoxon two-sample test. For this, PROC NPAR1WAY was used with medians and interquartile intervals. The results were considered significant with $P<0.05$.

Table 1. Variables investigated in mediastinoscopy and necropsy, which were classified as present or absent with scores 1 or 2 respectively.

\begin{tabular}{ccc}
\hline Variable & Classification & Score \\
\hline Tracheal visualization & Present & 1 \\
\hline RLN injury & Absent & 2 \\
\hline Traumatic esophageal & Present & 1 \\
injury & Absent & 2 \\
\hline Vascular injury & Present & 1 \\
\hline Tracheal bifurcation & Absent & 2 \\
visualization & Present & 1 \\
\hline Mediastinal integrity & Absent & 2 \\
\hline Location of prosthesis & Present & 1 \\
\hline & Absent & 2 \\
\hline
\end{tabular}

\section{RESULTS}

The results of necropsy and mediastinoscopy did not present significant differences at $P<0.05$. During the examinations, the presence of some mediastinal visceral lesions caused by the prosthesis, the integrity of the mediastinum and possible lesions to RLN and blood vessels (BV) were analyzed. We also investigated the location of the distal part of the prosthesis in the thoracic segment of the trachea and its dissection.

During the dissection, no difficulty was found in performing the technique. In some CCs there was some difficulty in the progression of the prosthesis through cranial mediastinum. This fact did not make prosthesis implantation impossible, which was verified by the two evaluation methods.

The nonparametric data of this experiment are organized in Table 2. The median, first quartile (Q1), third quartile (Q3), interquartile range (IQR), variance and number of specimens in which there were lesions were calculated. The last was expressed in number (n) and percentage (\%). To evaluate the technique, statistical comparison was made between mediastinoscopy and necropsy findings. The data were compared by the Wilcoxon test at 5\% probability. The median and interquartile ranges for the nonparametric evaluations are shown in Table 3.

The tracheas of all CCs were efficiently dissected, but in some cases problems that can happen during the procedure were noticed. This was checked by mediastinoscopy and confirmed by necropsy. The median of the scores was 1 (good dissection and visualization), with variance of zero for mediastinoscopy and 0.14 for necropsy. Two CCs had RLN involvement, with a median score of 2 (no nerve damage), with variance of 0.24 for the two methods used. In the evaluation of the blood vessels, the two methods had the same median value of 2 (absence of vascular involvement) and variance of 0.14 in mediastinoscopy, whereas by necropsy the variance was zero.

In the mediastinoscopy of one $\mathrm{CC}$, the prosthesis crossed the tracheal bifurcation, involving the posterior vessels of this region, which was confirmed by necropsy. However, the dissection showed no vascular lesions. The integrity of the mediastinum was assessed only by mediastinoscopy. There was no impairment caused by any of the procedures performed. This resulted in a median score of 1 (integrity) with zero variance. 
Incarceration of the esophagus by the prosthesis was visualized in one $\mathrm{CC}$, resulting in a median value of 2 (no impairment) and variance of 0.14 .

In $3 \mathrm{CCs}$ the bifurcation region was not well characterized during the endoscopic evaluation. During necropsy, it was possible to note that the dissection reached the site in question, but it was not possible to distinguish it from the surrounding tissue. The bifurcation of the trachea had a median value of 1 for both methods. However, the variance for mediastinoscopy was 0.24 , whereas for necropsy it was zero.
When the positioning of the prosthesis was evaluated in relation to the tracheal bifurcation, identical values were obtained by both methods. The median score was 1 , with variance of 0.62 . These values were obtained in specimens of $2 \mathrm{CCs}$, in which the prosthesis was cranial to bifurcation, while in $1 \mathrm{CC}$ the prosthesis extended beyond the tracheal bifurcation.

When comparing the medians and the interquartile ranges of the mediastinoscopy and necropsy by the Wilcoxon exact test, with 5\% significance, there was no significant difference between the evaluations by these 2 methods.

Table 2. Descriptive analysis of nonparametric data obtained by radiographic analysis, mediastinoscopy and necropsy.

\begin{tabular}{cccccccc}
\hline Variable & N & Med & Q1 & Q3 & IQR & Variance & Injury N $(\%)$ \\
\hline Tracheal visualization & 7 & 1 & 1 & 1 & 0 & 0.00 & $0(0)$ \\
NLR injury & 7 & 2 & 1 & 2 & 1 & 0.24 & $2(28.57)$ \\
BV injury & 7 & 2 & 2 & 2 & 0 & 0.14 & $1(14.29)$ \\
Tracheal bifurcation visualization & 7 & 1 & 1 & 2 & 1 & 0.24 & $2(28.57)$ \\
Mediastinal integrity & 7 & 1 & 1 & 1 & 0 & 0.00 & $0(0)$ \\
Traumatic esophageal injury & 7 & 2 & 2 & 2 & 0 & 0.14 & $1(14.29)$ \\
Location of prosthesis & 7 & 1 & 1 & 2 & 1 & 0.62 & - \\
\hline Tracheal visualization & 7 & 1 & 1 & 1 & 0 & 0.14 & $1(14.29)$ \\
NLR injury & 7 & 2 & 1 & 2 & 1 & 0.24 & $2(28.57)$ \\
BV injury & 7 & 2 & 2 & 2 & 0 & 0.00 & $0(0)$ \\
Tracheal bifurcation visualization & 7 & 1 & 1 & 1 & 0 & 0.00 & $0(0)$ \\
Traumatic esophageal injury & 7 & 2 & 2 & 2 & 0 & 0.14 & $1(14.29)$ \\
Location of prosthesis & 7 & 1 & 1 & 2 & 1 & 0.62 & - \\
\hline
\end{tabular}

$\mathrm{N}$ : number of specimens; $\mathrm{n}$ : CC numbers with some impairment; (\%): percentage of CCs with injury; Med: Median; IQR: interquartile range; Q1: first quartile; Q3: third quartile; NLR injury: impairment of at least one recurrent laryngeal nerve; BV injury: involvement of an important blood vessel in the mediastinum; Traumatic esophageal injury: esophageal impairment.

Table 3. Evaluation of the implantation technique of the intrathoracic helicoidal prosthesis by mediastinoscopy and necropsy.

\begin{tabular}{cccc}
\hline Variable & Mediastinoscopy & Necropsy & $P$-value* \\
\cline { 2 - 3 } Tracheal visualization & Med $\pm \mathrm{IQR}$ & Med $\pm \mathrm{IQR}$ & 0.05 \\
NLR injury & $1 \pm 0$ & $1 \pm 0$ & 0.05 \\
BV injury & $2 \pm 1$ & $2 \pm 1$ & 0.05 \\
Tracheal bifurcation visualization & $2 \pm 0$ & $1 \pm 0$ & 0.05 \\
Mediastinal integrity & $1 \pm 1$ & - & 0.05 \\
Traumatic esophageal injury & $1 \pm 0$ & $2 \pm 0$ & 0.05 \\
Location of prosthesis & $2 \pm 0$ & $1 \pm 1$ & 0.05 \\
\hline
\end{tabular}

Med: Median; IQR: interquartile range; NLR injury: involvement of at least one recurrent laryngeal nerve; BV injury: involvement of an important blood vessel in the mediastinum; Traumatic esophageal lesion: esophageal involvement. *Significant at 0.05 probability by the exact Wilcoxon two-sample test. 


\section{DISCUSSION}

In our study, the placement of the extraluminal prosthesis in the thoracic tracheal segment through cervical access, followed by mediastinoscopy and necropsy, allowed evaluation and classification of the different degrees of impairment of peri-tracheal structures, as well as validation of the efficacy of mediastinoscopy during the procedure.

Mediastinoscopy as an evaluation procedure can pose some complications [2]. However, it is a safe method with minimal invasion for investigation and treatment of mediastinal diseases [11,16,18]. Regarding the trachea, dissection was efficient in all CCs. The median of the scores was 1 (good dissection and visualization), with variance of zero for mediastinoscopy and 0.14 for necropsy. This variance for necropsy occurred due to the persistence of adhesions in the manubrium region in one of the CCs. In the region where the defective dissection occurred, the trachea was dorsal at the point of insertion of the optic, so this site became a "blind spot" in the endoscopic evaluation. For the evaluation of the blood vessels, the 2 methods had the same median value of 2 (absence of vascular involvement) and variance of 0.14 in mediastinoscopy, whereas by necropsy the variance was zero. This demonstrates the suitability of the technique and its low invasiveness and low possibility of traumatic injury to the adjacent structures of the trachea [8]. A recent study describes controlling hemostasis as the key to better visualization by the surgeon and reduction of the risk of iatrogenic nerve damage, especially the nerves in the area of the thorax entrance. The use of mediastinoscopy allows maintaining the endoscopic view of the mediastinal structures adjacent to the thoracic trachea, thereby minimizing injuries to the adjacent structures [12].

In this experiment, the entire tracheal circumference was dissected to allow implantation of the prosthesis and was performed non traumatically due to the low invasiveness, preserving important structures and mitigating possible complications $[10,17]$.

Two CCs had RLN involvement (28.57\%). This complication, although undesired, occurs due to the proximity of the RLN to the trachea $[3,12]$, and the risk of nerve injury can occur without the involvement of the prosthesis [2]. Thus, it is a complication that must be considered, which imposes the need for careful dissection. We reached a median score of 2 (no nerve damage), with variance of 0.24 , for the 2 methods used. Laryngeal paralysis is an important complication related to injury to the RLN during tracheal dissection for the implantation of the extraluminal prosthesis and placement of the ring, occurring in $11 \%$ to $21 \%$ of cases [3,14]. Some studies have described the association of a prophylactic technique for laryngeal paralysis, combining lateralization of the arytenoid with implantation of extraluminal prostheses, since concerns about this complication are associated with the potential risk to life. On the other hand, we believe that a complete postoperative evaluation can direct patients with this change in the immediate postoperative period, with arytenoid lateralization being performed only in patients with indication [3]. In a study in which 54 dogs received an extraluminal tracheal prosthesis, only $2 \%$ had unilateral laryngeal paralysis in the postoperative period, and this was corrected in a second surgical procedure using the arytenoid technique, with lateralization and consequent clinical improvement of the patient [12].

In the mediastinoscopy of $1 \mathrm{CC}$, the prosthesis crossed the tracheal bifurcation, involving the posterior vessels of this region, which was confirmed by necropsy. However, the dissection showed no vascular lesions. The mediastinal region is anatomically hard to access due to the large number of important small structures present [6,7]. Therefore, it is necessary for the surgeon to have experience in accessing and manipulating this region $[11,16]$. This result shows that the technique is not highly traumatic and can be successfully performed even though it is applied in the region of the mediastinum.

The integrity of the mediastinum was assessed only by mediastinoscopy. There was no impairment in any of the procedures performed. This resulted in a median score of 1 (integrity) with zero variance. Reports demonstrate that mediastinal rupture results in iatrogenic pneumothorax, which in most cases is treatable with thoracic drainage [2]. In this experiment, no rupture of the mediastinum was detected, demonstrating the adequacy of the proposed technique and its low invasiveness. The mediastinal pleura of the dog is not complete, and $\mathrm{CO}_{2}$ can pass from the mediastinal space to the right and/or left pleural space [18]. This is an important consideration, since 
it can lead to intraoperative pneumothorax and then to airway hypertension during ventilation. In our study, the procedure was performed on cadavers, but a future study might suggest in vivo by pneumatic mediastinoscopy suggests postoperative thoracentesis. The bifurcation of the trachea had a median value of 1 for both methods. However, the variance for mediastinoscopy was 0.24 , whereas for necropsy it was zero. In $3 \mathrm{CCs}$, the bifurcation region was not well characterized during the endoscopic evaluation. During necropsy, it was possible to note that the dissection reached the site in question, but it was not possible to distinguish it from the surrounding tissue. The azygos arch, bronchial artery, heart plexus and lung plexus of vagus nerve are preserved, so the incidence of postoperative cardiopulmonary complications through transmediastinal access is minimized, thus expanding the indications for surgery, especially for elderly patients and patients with cardiopulmonary comorbidities [18]. These observations were proven by the procedures performed in this experiment.

The insufflation of $\mathrm{CO}_{2}$ into the mediastinum enlarged the surgical space, which was helpful to fully expose the thoracic trachea and the adjacent structures such as the esophagus and RLN. But with the pressure caused by the pneumediastinum, we observed inversion of the tracheal muscle and temporary reduction in the tracheal diameter.

The esophagus is an important organ located near the trachea along its entire extension, and the injection of $\mathrm{CO} 2$ into the mediastinum makes the surgical field clearer, thus making the separation of esophagus easier and $[8,18]$. Hence, caution is necessary to avoid compromising this organ. In this study, $1 \mathrm{CC}$ had esophagus encased by the prostheses. This was confirmed by mediastinoscopy and necropsy. This resulted in a median value of 2 (no impairment) and variance of 0.14 .

When the positioning of the prosthesis was evaluated in relation to the tracheal bifurcation, identical values were obtained by both methods. The median score was 1 , with variance of 0.62 . These values were obtained in $2 \mathrm{CCs}$, in which the prosthesis was cranial to bifurcation, while in $1 \mathrm{CC}$ it was caudal. Despite this abnormal and undesired position in these cases, we believe that because the prosthesis is positioned covering a large portion of the trachea, this fact will not represent an additional risk factor, and that it can be solved with adequate measurement of the prosthesis and thoracic trachea, as well as with the appropriate metric graduation of the prosthesis in question.

The surgery by pneumatic mediastinoscopy is more difficult than thoracoscopy, since the medial thoracic entry to the sternum is very narrow, which causes the endoscopic instruments to clump and interfere with each other in the mediastinum [18]. Another factor is the limited mediastinal space, which is smaller than the thoracic cavity, so a small hemorrhage during the operation can make the anatomical space unclear, resulting in operational difficulties. These considerations are important, but by using technique proposed in this study, these factors are minimized due to the ease of technical implantation of the extraluminal tracheal stent, without the need for several instruments competing in the inflated mediastinal space.

No statistical differences were found between the 2 evaluation methods for the analyzed variables. This demonstrates the strong potential of the proposed evaluation techniques.

\section{CONCLUSION}

The implantation of the prosthesis in the thoracic trachea is possible by the cervical route, using atraumatic instruments for dissection and digital guidance through the median opening in the cranial thoracic region. Pneumatic mediastinoscopy was efficient during the implantation procedure. It is possible to evaluate the dissection of the trachea, to enable prevention or identify possible complications such as hemorrhage or injury of the RLN and pneumothorax, as well as to confirm the proper positioning of the implant at the end of the procedure.

Funding. This work was financially supported through grants from the CAPES (Coordination of Improvement of Higher Education Personnel) and Animal Science Graduate Program of the Darcy Ribeiro North Fluminense State University. The funders had no role in the design of the study, data collection, analysis and interpretation of data, or writing of the manuscript.

Ethical approval. The present study was submitted to the Animal Use Ethics Committee (CEUA) of the Northern Fluminense State University Darcy Ribeiro. The experiment was conducted in full compliance with all applicable research ethics and animal welfare regulations developed by The National Animal Experimentation Control Board (CONCEA).

Declaration of interest. The authors report no conflicts of interest. The authors alone are responsible for the content and writing of paper. 


\section{REFERENCES}

1 Becker W.M., Beal M., Stanley B.J. \& Hauptman J.G. 2012. Survival after surgery for tracheal collapse and the effect of intrathoracic collapse on survival. Veterinary Surgery. 41(4): 501-506.

2 Beteg F., Muste A. \& Lacatus R. 2010. Mediastinotomy by paratracheal approach in the dog - a preliminary report. Lucrari Stiintifice Medicina Veterinaria. 43(2): 90-94.

3 Chisnell H.K. \& Pardo A.D. 2014. Long-term outcome, complications and disease progression in 23 dogs after placement of tracheal ring prostheses for treatment of extrathoracic tracheal collapse. Veterinary Surgery. 44(1): 103-113.

4 Durant A.M., Sura P., Rohrbach B. \& Bohling M.W. 2012. Use of nitinol stents for end-stage tracheal collapse in dogs. Veterinary Surgery. 41(7): 807-817.

5 Freitag L. \& Darwiche K. 2014. Endoscopic treatment of tracheal stenosis. Thoracic Surgery Clinics. 24(1): 27-40.

6 Fujiwara H., Shiozaki A., Konishi H., Kosuga T., Komatsu S., Ichikawa D., Okamoto K. \& Otsuji E. 2015. Single-Port Mediastinoscopic Lymphadenectomy Along the Left Recurrent Laryngeal Nerve. The Annals of Thoracic Surgery. 100(3): 1115-1117.

7 Jin Y., Lu X., Xue L. \& Zhao X. 2018. Retrospective Comparison of Two Minimally Invasive Esophagectomy in the Treatment of Esophageal Cancer: Pneumatic Mediastinoscopy Versus Thoracoscopy. Journal of Laparoendoscopic and Advanced Surgical Techniques and Videoscopy. 29(5): 638-642.

8 Oshikiri T., Takiguchi G., Miura S., Takase N., Hasegawa H., Yamamoto M., Kanaji S., Yamashita K., Matsuda Y., Matsuda T., Nakamura T., Suzuki S. \& Kakeji Y. 2018. Current status of minimally invasive esophagectomy for esophageal cancer: Is it truly less invasive? Annals Gastroenterological Surgery. 3(2): 138-145.

9 Ranu H., Evans J., Sheth A. \& Madden P.B. 2010. Removal of Long-Term Tracheal Stents with Excellent Tracheal Healing. The Annals of Thoracic Surgery. 89(2): 598-599.

10 Raske M., Weisse C. \& Berent A.C. 2018. Immediate, short-, and long-term changes in tracheal stent diameter, length, and positioning after placement in dogs with tracheal collapse syndrome. Journal of Veterinary Internal Medicine. 32(2): 782-791.

11 Shrager J.B. 2010. Mediastinoscopy: still the gold standard. The Annals of Thoracic Surgery. 89(6): 2084-2089.

12 Suematsu M., Suematsu H., Minamoto T., Machida N., Hirao D. \& Fujiki M. 2019. Long-term outcomes of 54 dogs with tracheal collapse treated with a continuous extraluminal tracheal prosthesis. Veterinary Surgery. 48(5): 825834.

13 Tappin S.W. 2016. Canine tracheal collapse. Journal of Small Animal Practice. 57(1): 9-17.

14 Tinga S., Mankin K.M.T., Peycke L.E. \& Cohen N.D. 2015. Comparison of outcome after use of extra-luminal rings and intra-luminal stents for treatment of tracheal collapse in dogs. Veterinary Surgery. 44(7): 858-865.

15 Weisse C., Berent A., Violette N., McDougall R. \& Lamb K. 2019. Short-, intermediate-, and long-term results for endoluminal stent placement in dogs with tracheal collapse. Journal of the American Veterinary Medical Association. 254(3): 380-392.

16 Venissac N., Pop D. \& Mouroux J. 2009. Video-assisted mediastinoscopy as a therapeutic tool. Surgical Endoscopy. 23(11): 2466-2472.

17 Violette N.P., Weisse C., Berent A.C. \& Lamb K.E. 2019. Correlations among tracheal dimensions, tracheal stent dimensions, and major complications after endoluminal stenting of tracheal collapse syndrome in dogs. Journal of Veterinary Internal Medicine. 33(5): 2209-2216.

18 Zhu S., Yu S. \& Liu F. 2020. Combined single-port transmediastinal and laparoscopic access with $\mathrm{CO}_{2}$ insufflation for esophageal resection: a case report on a canine model. AME Case Report. 4: 6. 